\title{
Whole Breast Irradiation
}

National Cancer Institute

\section{Source}

National Cancer Institute. Whole Breast Irradiation. NCI Thesaurus. Code C116440.

External beam radiation that treats the entire breast. 